\title{
The Design of Bus Rapid Transit Routes in Cotonou based on Ant Colony Optimization Algorithm
}

\author{
Fousseni Gomina Mama \\ Transportation and Management College \\ Dalian Maritime University \\ Dalian, China \\ E-mail: gominafousseni@gmail.com
}

\author{
Zhongzhen Yang \\ Transportation and Management College \\ Dalian Maritime University \\ Dalian, China \\ E-mail: yangzhongzhen@263.com
}

\author{
Tetteh Evans Ago \\ Transportation and Management College \\ Dalian Maritime University \\ Dalian, China \\ E-mail: evansleeson@yahoo.co.uk
}

\begin{abstract}
The bus rapid transit (BRT) system is an efficient public transport mode implemented recently in many cities, but still unknown in many developing countries. Even though, the strategies developed in industrialized countries to enhance the viability of transportation systems cannot be unilaterally transferred to developing countries, the aim of this paper is to introduce the BRT concept in Cotonou by developing a new model. Based on ant colony optimization (ACO), a heuristic algorithm is developed to design and optimize the BRT routes (i.e. maximize direct-through travelers on unit length of BRT route), since the problem is NP-hard. With a numerical test from the case study, the effectiveness of the model and the algorithm is examined. Applying the model to the case of Cotonou, in Benin, we wish to stimulate the debate on the implementation of Bus Rapid Transit in developing cities because transportation policies and huge financial investments for inadequate road infrastructures and public bus system seem insufficient to solve the issue of urban mobility and its sustainability.
\end{abstract}

Keywords- BRT; optimization; ant colony optimization; public transport; Cotonou

\section{INTRODUCTION}

With rapid urbanization and economic growth, motorization has been accelerating in cities in developing countries. In Cotonou, the metropolitan city of Benin in the western African region, the number of motor vehicles per one thousand people has more than tripled in the past 20 years. Owning a private car or a motorized two-wheeler is a major aspiration for people in the city, where public transport service is often inadequate.

Unfortunately, central government and city managers in Cotonou are following the same caroriented transport development patterns made by many cities in developed countries in the past. Ironically, many cities in developed countries are now trying to recover from a car-dominated development era by halting the building of more infrastructures for private vehicles and re-allocating road space for public transport and non-motorized transport. From this approach, emerged a concept of BRT, already successfully implemented in many developed countries but it is still unknown in Cotonou where the problem is occurred.

Bus Rapid Transit (BRT) is an innovative transportation system, which combines the quality of rail transit and the flexibility of buses. BRT often operates on exclusive transit ways with high-capacity and high-speed vehicles. Since many features of BRT are similar to light rail transit (LRT), BRT may be considered to be able to improve transit service through incorporating favorable operational characteristics of rail-based transit systems. Referring to rail transit route planning, BRT routes need 
to cover large transit demand corridors which can provide enough travelers.

Planning BRT routes is similar with arterial transit route network design. Transit network design has been an important problem in urban transportation planning. There have been many successful studies on transit network planning over the past several decades. Ceder and Wilson (1986) introduced three-step method (trip assignment, planning routes and departure interval) into the design of network of public bus routes. Hasselstrőm (1981) presented a two-step model to optimize routes and frequency simultaneously. Wang (2001) developed a model aiming to maximize direct travelers. Yang et al. (2007) presented an optimization method of public bus network, which can efficiently obtain the trade-off between route length and the number of direct travelers and improve the efficiency of optimization. Recently, some studies have been completed on BRT route planning. Bai et al. (2007) optimized the BRT routes using tabu $\mathrm{k}_{\mathrm{k}}$ search algorithm and tested the model and the algorithm by simulation. Fan and Pan (2007) study the application of genetic algorithm on intelligent transport systems scheduling of vehicles. Sun et al. (2007) focus on BRT headway and scheduling combination optimization with genetic algorithm. An et al. (2008) employed particle swarm optimization to solve a set of bi-level programming models to find a feasible solution for the BRT network design problem. All the aforementioned studies stimulated us to develop a heuristic algorithm to optimize the routes of BRT.

The purpose of this paper is to extend the model of Yang et al. (2007) to design BRT routes in Cotonou. The objective of this study aims to maximize direct transit trip density, which is related to the direct transit demands and the length of the route. Some network optimization problems are NP-hard, which is difficult to be solved using traditional techniques. In this paper, ant colony algorithm is used to solve the optimization model.

\section{OPTIMIZATION MODEL}

Among existing studies, the direct traveler approaches are practical and convenient. They aim to maximize the number of the direct travelers under the length constraint or set the route along the shortest path. Maximization of the number of direct travelers may cause the bus routes in the network to be extremely long, whereas the latter limits the number of alternative routes and then damages the optimizing quality. Moreover, in both cases mentioned above, the route directions might not coincide with travelers flow directions. To overcome these problems, we choose the maximum direct traveler density as the optimal objective, that is, the maximum number of travelers per unit length. As a bus route is composed of the stops and the road segments it covers, the optimization problem is hereby equivalent to finding the corresponding stops and road segments. Here, $N$ denotes the stop collection; and $A$ denotes the collection of road segments. All of the alternative bus routes, the sub collections of $A$, are denoted as $\mathrm{S}=\{\mathrm{SOD}\}$. Then, if not considering the loop route, the bus network optimization model is as follows:

$$
\begin{array}{ll}
\max & D_{o d}=\frac{P_{o d}}{L_{o d}} \\
\text { s.t. } & \left\{\begin{array}{l}
L_{\text {min }} \leq L_{o d} \leq L_{\text {max }} \\
o d \in F \\
q_{o d}^{x} \leq 1.5 \\
m \neq n \quad \forall m, n \in S_{o d} \\
\forall l_{g h}>0.8 \mathrm{~km}
\end{array}\right.
\end{array}
$$

Where

$$
x_{i j}= \begin{cases}1 & \mathrm{i}, \mathrm{j} \text { are consecutive on Planning BRT routes; } \\ 0 & \text { else. }\end{cases}
$$

$D_{o d}=$ direct traveler density of the route starting from $O$ and ending at $D$;

$o=$ origin stops;

$d=$ destination stops;

$F=$ collection of origin and destination stops;

$\mathrm{P}_{\mathrm{ij}}=$ the number of direct travelers between stops $i$ and $j$

$P_{o d}=\sum_{g \in N} \sum_{h \in N} p_{g h} x_{g h}$ $=$ the number of direct travelers from original stop $i$ to destination stop $j$;

$l_{g h}=$ length of the road section $\alpha$ between $g$ and $h$, usually $l_{g h}=0.8 \sim 1.2 \mathrm{~km}$;

$L_{o d}=\sum_{g \in N} \sum_{h \in N} \Delta_{g h} l_{g h} x_{g h}$ $=$ if the length of BRT routes is too short, it will add the turnover time and affect the speed advantage of BRT. Hence, the length constraint of BRT routes is relaxed as $10 \mathrm{~km} \leq L_{\text {od }} \leq 25 \mathrm{~km}$;

$\Delta_{g h}= \begin{cases}1 & \mathrm{~g}, \mathrm{~h} \text { are consecutive on Planning BRT routes; } \\ 0 & \text { else. }\end{cases}$ $q_{o d}^{x}=$ nonlinear rate, because usually buses with large capacity is adopted in BRT routes, if the value of nonlinear rate is too large, the operational speed will be affected. 
$m, n=$ the middle stops of candidate routes. This constraint aims to control the route trend, which means one route should pass through the one stop twice or more.

\section{ALGORITHM DESIGN}

\section{A. Ant Colony Optimization Introduction}

The ant colony optimization (ACO) algorithm is a probabilistic technique for solving computational problems which can be reduced to finding good paths through graphs. ACO was firstly introduced by Dorigo (1992) and has been used to find the solutions for many difficult combinatorial optimization problems. In ACO, artificial ants build solutions by moving on the problem graph. During the process they deposit artificial pheromone on the graph in such a way that future artificial ants can build better solutions. Due to positive feedback rule, the ACO possesses powerful ability in searching better solutions for combinatorial optimization problems.

\section{B. Transition Rules}

The moving of ants is according to certain rules instead of unconscious. First, ants can only move through feasible neighbor nodes, which suggest that many nodes are unavailable for the ant moving. For example, the nodes which have been visited are unavailable in the optimization problem of BRT routes. The transition rules are as follow: the ant chooses available nodes according to the probability of each available node. The method of choosing probability of node $j$ for the ant $k$ at the node $i$ is as follows:

$$
P_{i j}(k)= \begin{cases}\frac{\tau_{i j}^{\alpha} \times \eta_{i j}^{\beta}}{\sum_{h \notin t a b u_{k}} \tau_{i h}^{\alpha} \times \eta_{i h}^{\beta}} & j \notin t a b u_{k} \\ 0 & \text { others }\end{cases}
$$

Here, $\tau$ : the intensity of which reflects the prior experience of the ants at this searching, and also means the total pheromone which cumulated during the running process of the ants; $\eta$ : visibility, the value of which is obtained by solving a greedy algorithm of an original problem; it only considers the local and encourages the ants to search for the local optimized routes; $\alpha, \beta$ : the pheromone heuristic factor and the visibility heuristic factor; $t a b u_{k}$ : the setting of unavailable nodes of the ant $k$.

\section{Pheromone Refreshing Strategy}

According to the transition rules, an ant can construct a solution of an original problem. For example, a solution means that the ant has searched a BRT route between original node and destination one. Then, the quantity of pheromone of each segment will be refreshed by the formula (3):

$$
\tau_{i j}(t+1)=\rho \times \tau_{i j}(t)+\sum_{k=1}^{p} \Delta \tau_{i j}^{k}
$$

Where $\rho$ is the remaining coefficient of pheromone $(0,1) ; p$ : the scale of ants; $\Delta \tau_{i j}^{k}:$ The pheromone increment when the ant $k$ is on line $(i, j)$ in one loop.

To improve the efficiency of updating pheromone, antweight strategy (Yang et al. ,2007) is used to compute the pheromone increment, which can integrate the global and local information of the search. Specifically, the updating strategy of the increased pheromone is as follow:

$$
\Delta \tau_{i j}^{k}=\left\{\begin{array}{cc}
\frac{Q}{f^{k}} \times \frac{f^{k}-f_{i j}}{(n-2) f^{k}} & \text { if } \operatorname{link}(i, j) \text { on the } k_{t h} \text { route } \\
0 & \text { otherwise }
\end{array}\right.
$$

Where $Q=$ a constant, $f^{k}=$ the direct traveler density of route $k, f_{i j}=$ the direct traveler density on link $(i, j), n=$ the number of the stops along route $k(n>2)$.

The strategy for updating the increased pheromone consists of two components: the first is global pheromone increment $Q / f^{k}$ related to the direct traveler density of route $k$; and the second one is local pheromone increment $\left(f^{k}-f_{i j}\right) /\left((n-2) f^{k}\right)$ based on the contribution of link $(i, j)$ to route $k$. Thus, the update strategy ensures that the assigned pheromone increments are directly proportional to the direct traveler density. The more favorable of the link/route, the more pheromone increment is allocated to, and the more accurate directive information is provided to the later search. Meanwhile, by adjusting the pheromone assigning method for the links of current optimal path automatically, the algorithm can facilitate more delicate searches in the next cycle in a more favorable area. This helps to improve the learning capacity of the algorithm from past searches, and enhance the efficiency.

\section{CASE STUDIES}

In order to examine the feasibility and validity of our model and algorithm, the urban zone of Cotonou, is used in our case study. Cotonou is the biggest city (about a million inhabitants for just $79 \mathrm{~km} 2$ ) and headquarters of main economic, institutional, commercial, financial and diplomatic centers of Benin Republic. Attractive because of the port, airport and job opportunities, Cotonou has a special case for being an economic capital with two wheels motorcycle called 'Zem" as a predominant mode of 
transport. The recent buses routes and several bus stops implemented by city authorities and managed by a private operator called Benafrique, failed to satisfy the citizens' expectation in term of transport. The preference is still giving to the paratransit usage.

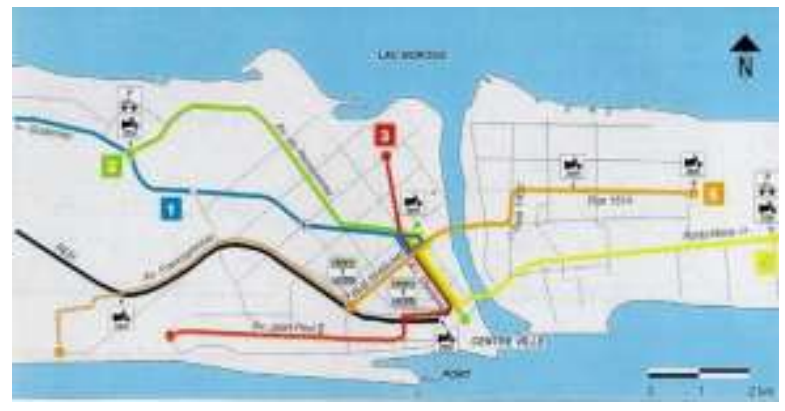

Figure 1. Map of public bus transport Network in Cotonou

Using Cotonou, as a case study, the purpose of this paper is to implement Ant Colony Optimization (ACO). Therefore, the Origin-Destination (OD) survey is conducted in the city and the OD Matrix is realized. Based on that, the ACO is applied to search the routes with largest direct traveler density for BRT routes. The result is shown in Figure 1.

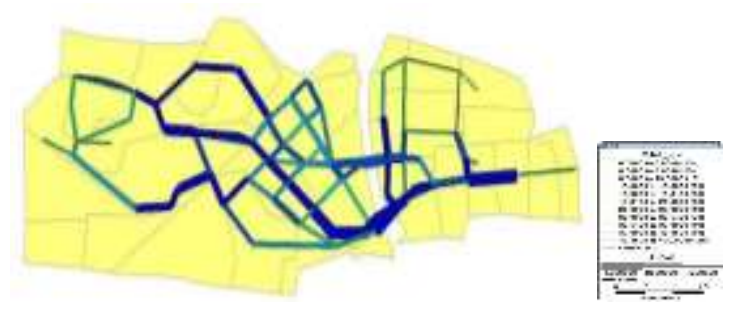

Figure 2. The Resulting BRT Routes

To validate the designed BRT routes, transit passenger assignment is implemented on the networks with or without BRT routes, respectively. The result is shown in Figures 2 and 3.

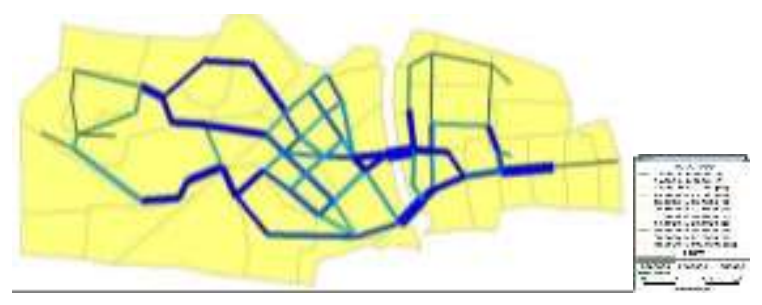

Figure 3. Distribution Result without BRT Routes

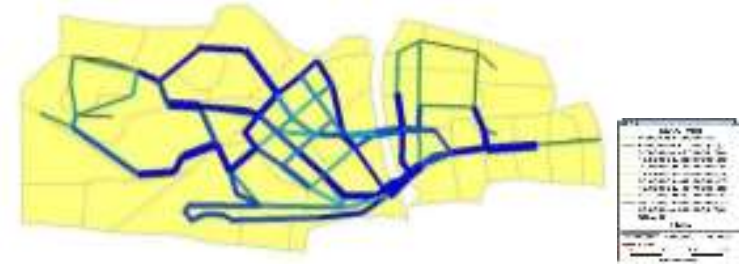

Figure 4. Distribution Result with BRT Routes

Because of its advantages such as the speediness, large capacity and so on, travelers flow is collected on the BRT route; and the flow of the bus routes near the BRT route is decreased significantly. It is necessary to change the public bus routes in the service area of BRT routes. With BRT routes, the total time of trip of residents will decrease. This is because that the BRT route is built along the public corridor, where the dense travelers flow is collected. Moreover, it employs public special bus lane to ensure fast operating speed.

The economic and financial sustainability of cities in both developed and developing countries are inextricably linked with the viability of the city's transportation system. Unfortunately elements of urban transportation systems in industrialized countries differ considerably from those of developing countries. Consequently, strategies developed in industrialized countries to enhance the viability of the transportation systems cannot be unilaterally transferred to developing countries including those in sub-Sahara Africa. Specifically, the central government and local authorities of Cotonou emphasized the need to provide policies geared towards effective, safe and reliable transportation services for citizens by considering the specific case of Cotonou, a city in which the two wheels motorcycles are the most captive transport mode. As for policy implications, although paratransit vehicles with small size, i.e., Zem, have significantly a negative impact, authors believe that it is still necessary to keep such paratransit with small size for a certain area and period from the perspective of supplying job opportunity and social stability. Certainly such paratransit with small size is suggested to be restricted in limited and predefined districts as main modes in very short distance and/or as access/egress modes in longer distance just from the perspective of reducing the traffic congestion and travel safety. Due to the prevalent problem of the budget limitation in Cotonou, it is almost impossible to cover the urban transportation network by mainly Bus Rapid Transit which is environment-friendly and efficient public transit. Naturally, the combination of Zem and BRT system will 
be a wise choice. More research must be done to deeply analyze and evaluate the efficiency of this suggestion.

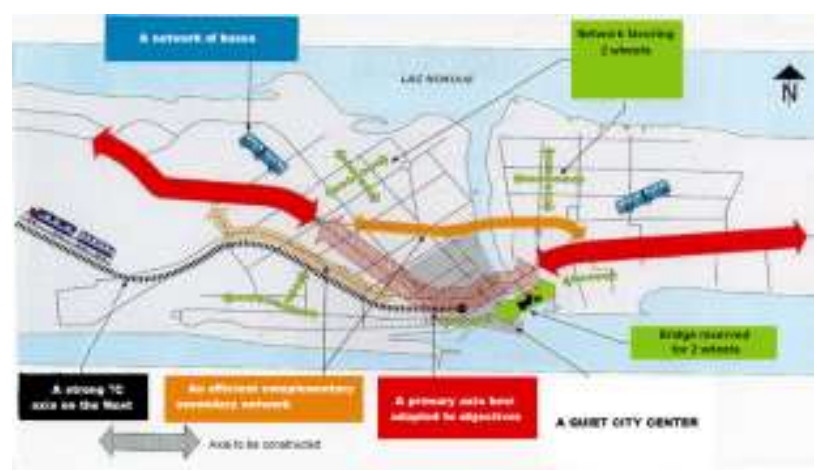

Figure 5. Plan of Urban mobility network in Cotonou combining different modes of transport.

\section{CONCLUSIONS}

High-quality transit service is considered as an effective way to attract travelers away from private car trips toward the transit use in congested metropolitan areas. Referring at many developed countries experience, this paper introduce the BRT system design in Cotonou and presents an optimization model of BRT routes which aims to maximize the density of direct travelers flow. This model has the objectives of minimizing transfer times and maximizing traveler flow of unit, by satisfying the constraints of the length of routes and nonlinear coefficient. An ACO based on the coarse-grain model is designed to solve the model. Finally the validity of the model and algorithm is examined in the case study. The result indicates that the model developed here can be used to optimize the routes of BRT effectively. Applying the model to the case of Cotonou, in Benin, the author want to stimulate the debate on the choice of strategies for sustainable transportation by accelerating the implementation of Bus Rapid Transit in developing countries where transportation policies and allocated investments for road infrastructures and public bus system, seem insufficient to solve the issue of urban mobility.

\section{REFERENCES}

[1] An, J., Teng J., and Meng L. Y. (2008), “A BRT Network Route Design Model." Proceeding of the $11^{\text {th }}$ International IEEE Conference on Intelligent Transportation Systems Beijing, China, October 12-15.

[2] Bai, Z. J. and He G. G. (2007). "Study on simulation optimization of bus rapid transit networks system based on Tabu search algorithm." Computer Engineering and Applications, 43( 17): 226229.

[3] Ceder A., and Wilson, N. H. M. (1986). "Bus network design." Transportation Research. Part B, 20B (4):331-44

[4] Dorigo, M. (1992), "Optimization, learning and natural algorithms." Politecnico di Milano, Italy.

[5] Fan, Q. S., and Pan, W. (2007). "Application research of genetic algorithm inintelligent transport systems scheduling of vehicle.' Computer and Digital Engineering, 35(5): 34-35.

[6] HasselstrÖm, D. (1981). "Public transportation planning - a mathematical programming approach." Göteborg: University of Göteborg.

[7] Sun, C. J., Zhou, W., and Wang, Y. Q. (2008), "Scheduling combination and headway optimization of bus rapid transit." Journal Of Transportation systems Engineering And Information Technology, 8(5), 61-67.

[8] Wang, W., Yang, X. M., and Chen, X W (2001). "Planning method and management technology of urban public transportation system.” Beijing: Science Press.

[9] Yang, Z. Z., Yu, B., and Cheng C. T (2007). "A parallel ant colony algorithm for bus network optimization." Computer-Aided Civil and Infrastructure Engineering, 22, 44-55.

[10] Spaethling D. Sustainable transport. The American experience. In: Proceedings of SeminarC.Planning for Sustainability of the 24th European Transport Forum. PTRC Education and Research Services Limited. London, England, September 1996.

[11] OECD. Towards Sustainable Transport. Paris: OECD Publications, 1996.

[12] Fousseni, G. (2013). Urban Transport Issues in Cotonou: Analysis and Perspectives, 10.4028/www. scientific.net/AMR.850-851.1118. 